\title{
The Topological Conditions: The Properties of the Pair of Conjugate Tress
}

\author{
Luis Hernandez-Martinez, Arturo Sarmiento-Reyes, Miguel A. Gutierrez de Anda
}

Electronics Department, National Institute for Astrophysics, Optics and Electronics, P.O. Puebla, Pue. Mexico. Email: \{luish,jarocho,mdeanda\}@inaoep.mx

Received November $29^{\text {th }}, 2009$; revised January $4^{\text {th }}, 2010$; accepted January $6^{\text {th }}, 2010$.

\begin{abstract}
This paper presents some important properties emanating from the pair of conjugate trees. The properties are obtained by resorting to the fundamental loops and cutsets in the circuit topology. The existence of such a pair is one of the conditions for a nonlinear resistive circuit to have one and only one DC solution.
\end{abstract}

Keywords: Pair of Conjugate Trees, Topological Conditions, Fundamental Cutsets and Loops, Analogue Circuits

\section{Introduction}

Graph Theory is used for the study of real-world Systems possessing a binary relation between elements of a certain set within the system description. Among other discipline (Circuit Theory) has received outstanding contributions from the study of graphs. Some of the contributions may be found in the solution to specific problems related to electrical network analysis, nonlinear circuit theory, circuit diagnosis and circuit synthesis [1,2].

A line of research developed in recent years has attempted to determine the relationships between the topology of a circuit and its functionality, which has derived in a deeper knowledge in the general problem of nonlinear circuits $[3,4]$. One important work has been reported in [5] and [6], where a topological criterion for the existence and uniqueness of the solution of linear circuits has been proposed. This criterion is based on two definitions of Graph Theory: the pair of conjugate trees and the uniform partial orientation of the resistors.

In this paper, the attention is focused on several properties of the pair of conjugate trees; these are highlighted by looking at the resulting loop and cutset matrices.

\section{Preliminary Considerations}

The scope of the work is restricted to certain types of basic circuit elements: resistors $(\mathrm{R})$, voltage sources $(\mathrm{V})$, current sources $(\mathrm{I})$, nullators $(\mathrm{O})$ and norators $(\mathrm{P})$. However, it must be emphasised that these circuit components are used to model the original circuit through an equivalent circuit denoted as the nonlinear resistive circuit struc -ture. Besides, an important condition concerning singular elements must be fulfilled: nullators and norators must appear in the circuit in equal numbers.

Other devices can be used by building up equivalent schemes consisting of models containing the set of basic elements. As an example, the Figure 1 shows the equivalent circuit of a bipolar transistor.

Herein, we retake two definitions from [3] in order to set up the further development of the diagnostic method:

\section{Definition 1}

A nonlinear resistive circuit structure is a graph whose branches are labeled with the following six element types.

- Independent voltage sources

- Independent current sources

- V-resistors (voltage controlled)

- I-resistors (current controlled)

- Nullators

- Norators

A circuit $\mathbf{C}$ has a structure $\mathbf{S}$ if the graph of $\mathbf{C}$ and $\mathbf{S}$ coincide and if $\mathbf{C}$ has elements of the type prescribed by $\mathbf{S}$ on each branch.

A nonlinear resistor can be substituted by one of the following equivalents:

1) The element is converted into a linear resistor if it is strictly increasing.

2) The element is converted into a voltage source if it is voltage-controlled.

3) The element is converted into a current source if it is current-controlled. As shown in the Figure 2. 


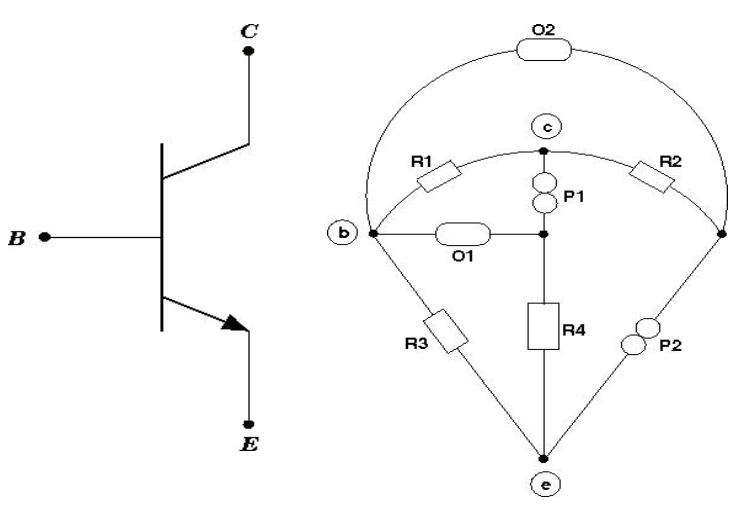

Figure 1. Equivalent circuito of a bipolar transistor

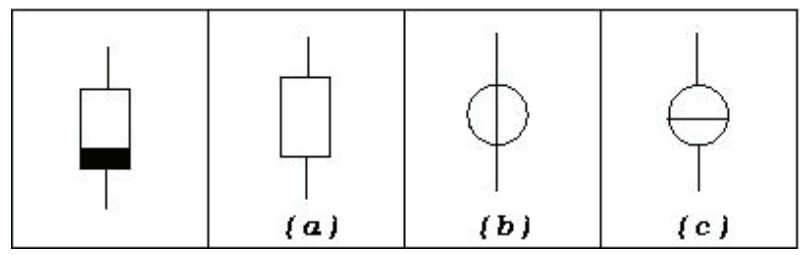

Figure 2. Equivalent of the nonlinear resistor

\section{Definition 2}

Two trees $t^{\prime}$ and $t^{\prime \prime}$ of a nonlinear resistive circuit structure constitute a pair of conjugate trees if:

- $t^{\prime}$ is composed of all norators, all voltage sources and a subset of the resistors, and

- $t^{\prime \prime}$ is composed of all nullators, all voltage sources and the same resistors as $\$ t^{\prime} \$$.

The subsets of the resistors may also be empty or contain all resistors.

The Figure 3 shows the pair of conjugate trees formed according to the Definition 2. On one hand, the tree $t^{\prime}$ is formed by the norators, both voltage sources and the resistor $R 1$, as given in the Figure 3(a). On the other hand, the tree $t^{\prime \prime}$ is formed by the nullator, both voltage sources and the same resistor as given in the Figure 3(b).

\section{Properties of the Pair $\left(t^{\prime}, t^{\prime \prime}\right)$}

From the definitions above, two associated graphs of the same linear structure can be derived, namely $g^{\prime}$ and $g^{\prime \prime}$. It yields:

$$
g^{\prime}=t^{\prime} \cup \tilde{t}_{c} \quad g^{\prime \prime}=t^{\prime} \cup \tilde{t}_{c}
$$

These graphs are depicted in Figure 4. However, it must be noticed that in fact, apart from the consideration of different trees, the relationship holds:

$$
g^{\prime}=g^{\prime \prime}
$$

As a result of the definitions given in Section 2, the pair of conjugate trees is formed as:

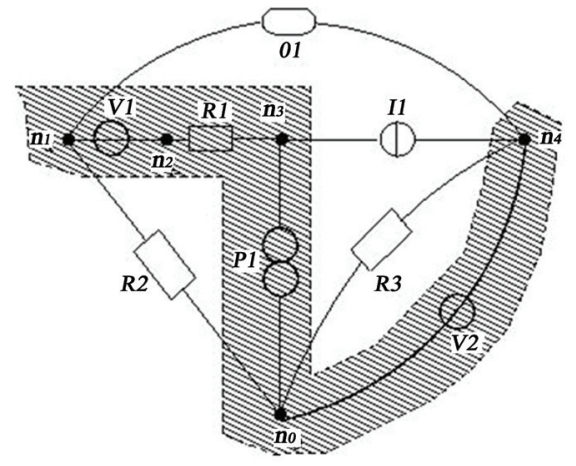

(a) Tree $t^{\prime}$

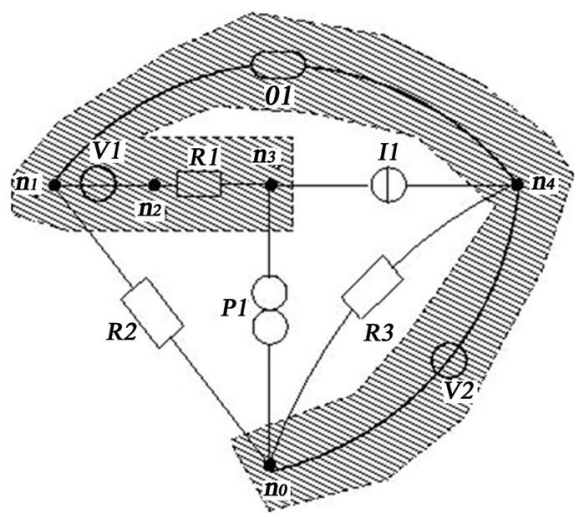

(b) Tree $t^{\prime \prime}$

Figure 3. The pair of conjugate trees
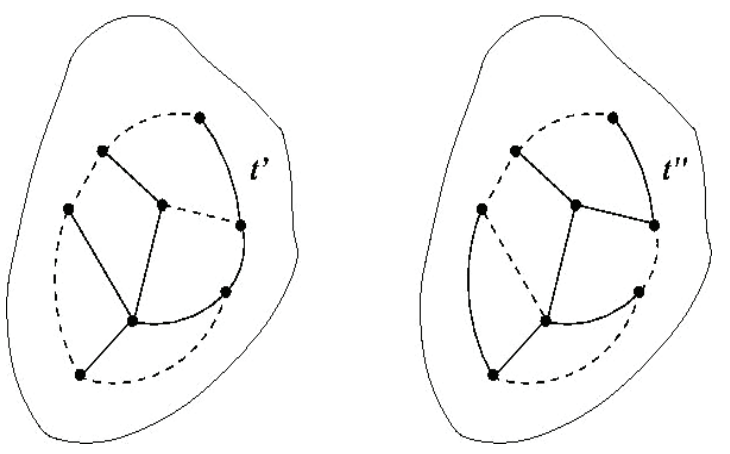

Figure 4. The graphs of a linear structure associated to the pair $\left(t^{\prime}, t^{\prime \prime}\right)$

$$
t=\{P, V, R\} \quad t=\left\{O, V, R_{a}\right\}
$$

where $P, O$, and $V$ are the branches of all norators, all nullators and all voltage sources respectively. In addition, $R_{a}$ is the set of branches of the common resistors $\in t^{\prime}$ and $t^{\prime \prime}$. Furthermore, two pairs of conjugate co-trees $(\tilde{t}, \tilde{t})$ arise. They are formed as:

$$
\tilde{t}=\left\{O, R_{b}\right\} \quad \tilde{t}=\left\{P, R_{b}\right\}
$$


where $R_{b}$ is the set of branches of those resistors not in $t^{\prime}$ nor in $t^{\prime \prime}$. It clearly results that the complete set of resistors $R$, is formed by:

$$
R=R_{a} \cup R_{b}
$$

Some dimensions may be mentioned now:

$$
\begin{aligned}
& p-\text { total number of norators } \\
& o-\text { total number of nullators } \\
& v-\text { total number of voltage sources } \\
& a-\text { total number of resistors } \in R_{a} \\
& b-\text { total number of resistors } \in R_{b} \\
& r-\text { total number of resistors } \in R \\
& \quad \text { i.e. } \quad r=a+b \\
& l-\text { total number of fundamental loop } \\
& c-\text { total number of cutsets } \\
& \quad \text { with the additional requirement } \\
& \quad \text { some variables must be defined: } \\
& u_{p}-\text { norators voltages } \\
& u_{o} \text { - nullator voltages } \\
& u_{v}-\text { voltage sources } \\
& u_{a}-\text { resistor voltages of } R_{a} \\
& u_{b} \text { - resistor voltages of } R_{b} \\
& i_{p}-\text { norators currents } \\
& i_{o}-\text { nullators currents } \\
& i_{v}-\text { current sources } \\
& i_{a}-\text { resistor currents of } R_{a} \\
& i_{b}-\text { resistor currents of } R_{b}
\end{aligned}
$$$$
l-\text { total number of fundamental loops }
$$$$
\text { with the additional requirement of } p=o \text {. Besides, }
$$
some variables must be defined:

\section{Properties}

Hereafter, the properties of the pair of conjugate trees $\left(t^{\prime}\right.$, $t^{\prime \prime}$ ) are obtained by resorting to the fundamental loops and fundamental cutsets of the associated graphs. Because only one branch of the co-tree may be present in a fundamental loop, the structure of the fundamental loop matrix has the form:

$$
C=\left[C_{T} \mid C_{L}\right]=\left[C_{T} \mid I_{L}\right]
$$

where $C_{T}$ is the tree-part of the fundamental loop matrix, and $C_{L}=I_{L}$ is the co-tree part. Because only one branch of the tree may be present in a fundamental cutset, the structure of the fundamental cutset matrix has the form:

$$
D=\left[D_{T} \mid D_{L}\right]=\left[I_{T} \mid D_{L}\right]
$$

where $D_{T}=I_{T}$ is the tree-part of the fundamental cutset matrix, and $D_{L}$ is the co-tree part. In the following, Kirchoff's Laws for both graphs are analyzed in order to determine the properties of the loops and cutsets.

\subsection{KVL for $g^{\prime}$}

Since the co-tree branch present in a fundamental loop must belong either to $O$ or $R_{b}$, then the matrix in Equation (6) can have the form:

$$
C=\left[\begin{array}{ll|ll}
C_{T} & I_{o} & \\
& & I_{b}
\end{array}\right]
$$

where $I_{o}$ and $I_{b}$ are identity matrices of order $o$ and $b$ respectively. Two types of loops arise:

$l_{o}^{\prime}-$ a loop in $g^{\prime}$ having a link

from $O$

$l_{b}^{\prime}$ - a loop in $g^{\prime}$ having a link

from $R_{b}$

Furthermore, $C_{T}$ can also be partitioned, which yields:

$$
C=\left[\begin{array}{lll|ll}
C_{o p} & C_{o v} & C_{o a} & I_{a} & \\
C_{b p} & C_{b v} & C_{b a} & & I_{b}
\end{array}\right]
$$

where every submatrix denoted as $C_{x y}$ is a matrix of size $\mathrm{x} \times \mathrm{y}$. Since $\mathrm{o}=\mathrm{p}, C_{o p}$ is a square matrix. Moreover, the size of $C^{\prime}{ }_{T}$ is $(o+b) \times(p+v+a)$. KVL is given as:

$$
C^{\prime} u=0
$$

The partitioning above allows us to establish KVL as:

$$
\left[\begin{array}{lll|l}
C_{o p} & C_{o v} & C_{o a} & I_{o} \\
C_{b p} & C_{b v} & C_{b a} & I_{b}
\end{array}\right]\left[\begin{array}{c}
u_{p} \\
u_{v} \\
\frac{u_{a}}{u_{o}} \\
u_{b}
\end{array}\right]=0
$$

which is a system of $(o+b)$ loop equations in $(p+v+a+o+b)$ branch voltages. For the nullors, $U_{o}=0$, then KVL can be re-written as:

$$
\left[\begin{array}{lll}
C_{o p} & C_{o v} & C_{o a} \\
C_{b p} & C_{b v} & C_{b a}
\end{array} \mid I_{b}\right]\left[\begin{array}{l}
u_{p} \\
u_{v} \\
\frac{u_{a}}{u_{b}}
\end{array}\right]=0
$$

\subsection{KCL for $g^{\prime}$}

Since the tree branch present in a fundamental cutset must belong either to $\mathbf{P}$ or $\mathbf{V}$ or $R_{a}$, then the matrix in Equation (7) can have the form:

$$
D=\left[\begin{array}{ccc|c}
I_{p} & & \\
& I_{v} & & D_{L} \\
& & I_{a} &
\end{array}\right]
$$

where $I_{p}, I_{v}$ and $I_{a}$ are identity matrices of order 
$\mathrm{p}, \quad v$ and $a$ respectively. Three types of cutsets arise:

$c^{\prime}{ }_{p}-$ a cutest in $g^{\prime}$ having a twig

from $P$

$c_{v}^{\prime}-$ a cutest in $g^{\prime}$ having a twig

from $V$

$c_{a}^{\prime}-$ a cutest in $g^{\prime}$ having a twig

from $R_{a}$

Furthermore, $D_{L}^{\prime}$ can also be partitioned, which yields:

$$
D=\left[\begin{array}{lll|ll}
I_{p} & & & D_{p o} & D_{p b} \\
& I_{v} & & D_{v o} & D_{v b} \\
& & I_{a} & D_{a o} & D_{a b}
\end{array}\right]
$$

where every submatrix denoted as $D_{x y}^{\prime}$ is a matrix of size $\mathrm{x} \times \mathrm{y}$. Since $o=p, D_{p o}$ is a square matrix. Moreover, the size of $D_{L}^{\prime}$ is $(p+v+a) \times(o+b) . \mathrm{KCL}$ is given as:

$$
D i=o
$$

The partitioning above allows us to establish KCL as:

$$
\left[\begin{array}{lll|ll}
I_{p} & & & D_{p a} & D_{p b} \\
& I_{v} & & D_{v o} & D_{v b} \\
& & I_{a} & D_{a o} & D_{a b}
\end{array}\right]\left[\begin{array}{c}
i_{p} \\
i_{v} \\
i_{a} \\
i_{o} \\
i_{b}
\end{array}\right]=0
$$

which is a system of $(p+v+b)$ cutset equations in $(p+v+a+o+b)$ branch currents. For the nullors, $i_{o}=0$, then KCL can be re-written as:

$$
\left[\begin{array}{lll|l|l}
I_{p} & & & D_{p b} \\
& I_{v} & & D_{v b} \\
& & I_{a} & D_{a b}
\end{array}\right]\left[\begin{array}{l}
i_{p} \\
i_{v} \\
i_{a} \\
i_{b}
\end{array}\right]=0
$$

Based on the orthogonality relationship:

$$
C_{T}=D_{T}^{T}
$$

where $\mathrm{D}_{\mathrm{T}}^{\prime \mathrm{T}}$ stands for the transpose of $\mathrm{D}_{\mathrm{T}}^{\prime}$ (see the Figure 5), the following equalities arise:

$$
\begin{array}{lll}
\mathrm{C}_{\mathrm{op}}^{\prime \mathrm{T}}=\mathrm{D}_{\mathrm{po}} & \mathrm{C}_{\mathrm{ov}}^{\prime \mathrm{T}}=\mathrm{D}_{\mathrm{vo}} & \mathrm{C}_{\mathrm{oa}}^{\prime \mathrm{T}}=\mathrm{D}_{\mathrm{av}}^{\prime} \\
\mathrm{C}_{\mathrm{bp}}^{\prime \mathrm{T}}=\mathrm{D}_{\mathrm{pb}}^{\prime} & \mathrm{C}_{\mathrm{bv}}^{\prime \mathrm{T}}=\mathrm{D}_{\mathrm{vb}}^{\prime} & \mathrm{C}_{\mathrm{ba}}^{\prime \mathrm{T}}=\mathrm{D}_{\mathrm{ab}}^{\prime}
\end{array}
$$

In order to illustrate the properties of the work, consider the linear circuit given in Figure 6, the fundamental loop and cutset matrices are given by:
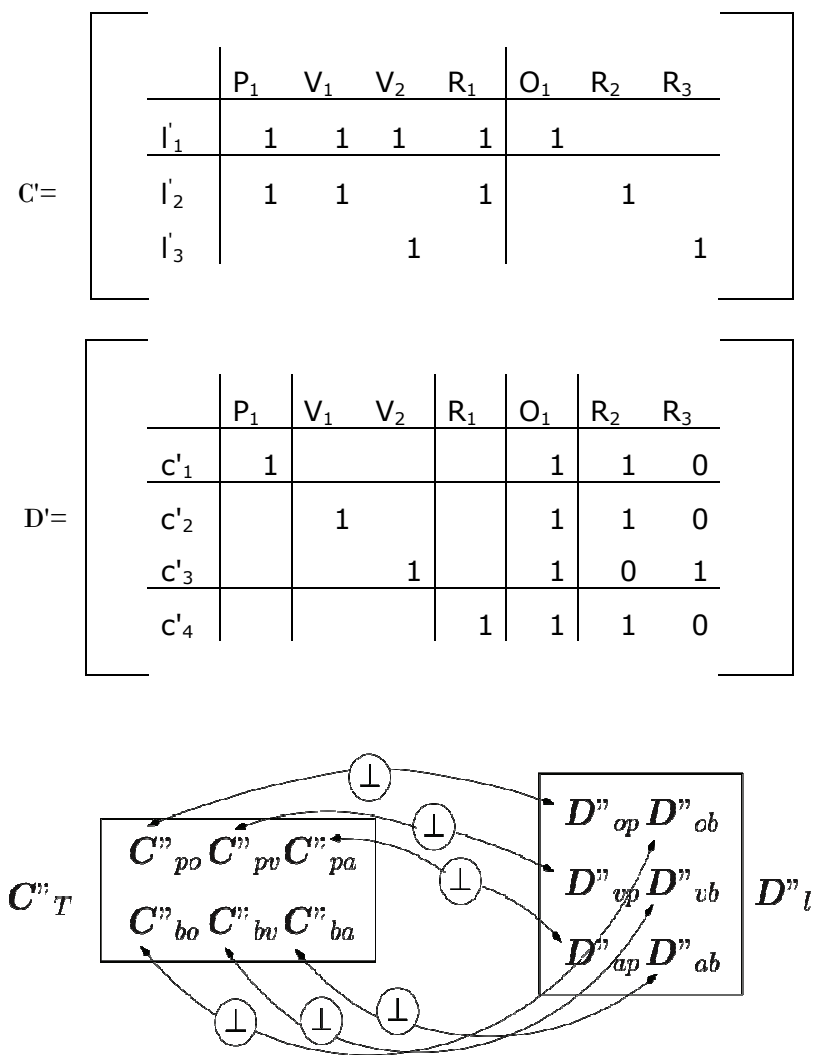

Figure 5. Orthogonality in $g^{\prime}$

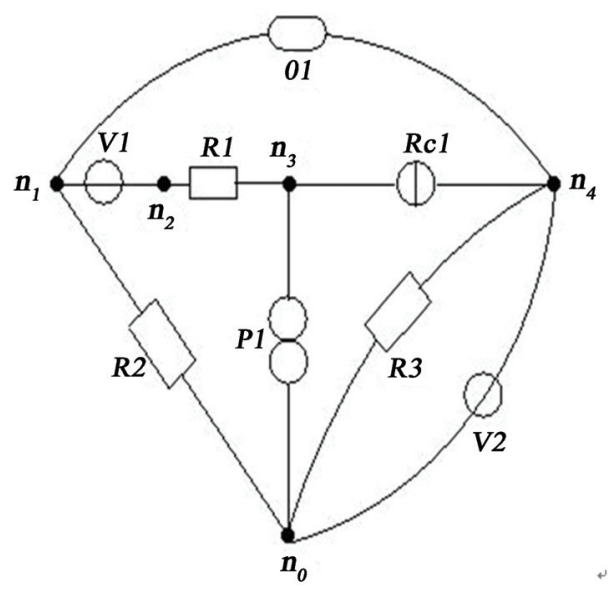

Figure 6. Case of study

where the columns have been labelled with the element names and the rows with the loop and cutsets respectively. The labels however, do not belong to the matrix.

\subsection{KVL for $g^{\prime \prime}$}

Since the co-tree branch present in a fundamental loop must belong either to $P$ or $R_{b}$, then the matrix in Equation (6) can have the form: 


$$
C^{\prime \prime}=\left[C_{T}^{\prime \prime} \mid \begin{array}{ll}
I_{p} & \\
& I_{b}
\end{array}\right]
$$

where $I_{p}$ and $I_{b}$ are identity matrices of order $P$ and $b$ respectively. Two types of loops arise:

$l_{p}^{\prime \prime}-$ a loop in $g^{\prime \prime}$ having a link

from $P$

$l_{b}^{\prime \prime}-$ a loop in $g^{\prime \prime}$ having a link

from $R_{b}$

Furthermore, $C_{T}^{\prime \prime}$ can also be partitioned:

$$
C^{\prime \prime}=\left[\begin{array}{lll|ll}
C_{p o}^{\prime \prime} & C_{p v}^{\prime \prime} & C_{p a}^{\prime \prime} & I_{p} & \\
C_{b o}^{\prime \prime} & C_{b v}^{\prime \prime} & C_{b a}^{\prime \prime} & & I_{b}
\end{array}\right]
$$

where every submatrix denoted as $C^{\prime \prime}{ }_{x y}$ is a matrix of size $x \times y$.

Since, $C^{\prime \prime}{ }_{p o}$ is a square matrix. Moreover, the size of $C^{\prime \prime}{ }_{T}$ is $(p+b) \times(o+v+a) . \mathrm{KVL}$ is given as:

$$
C^{\prime \prime} u=0
$$

The partitioning above allows us to establish KVL as:

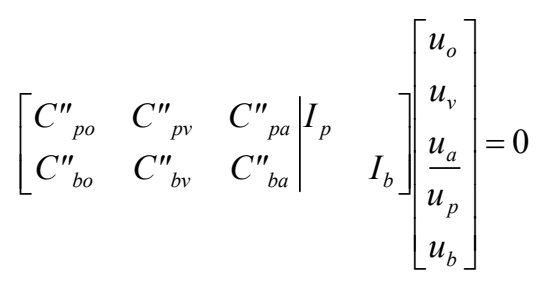

which is a system of $(o+b)$ loop equations in $(o+v+a+p+b)$ branch voltages. For the nullors, $u_{o}=0$, then KVL can be re-written as:

$$
\left[\begin{array}{ll|l}
C^{\prime \prime}{ }_{p v} & C^{\prime \prime}{ }_{p a} & I_{p} \\
C^{\prime \prime}{ }_{b v} & C^{\prime \prime}{ }_{b a} & \\
& I_{b}
\end{array}\right]\left[\begin{array}{l}
u_{v} \\
\frac{u_{a}}{u_{p}} \\
u_{b}
\end{array}\right]=0
$$

\subsection{KCL for $g^{\prime \prime}$}

Since the tree branch present in a fundamental cutset must belong either to $O$ or $V$ or $R_{a}$, then the matrix in Equation (7) can have the form:

$$
D^{\prime \prime}=\left[\begin{array}{lll|l}
I_{o} & & & \\
& I_{v} & & D^{\prime \prime}{ }_{L} \\
& & I_{a} &
\end{array}\right]
$$

where $I_{o}, I_{v}$ and $I_{a}$ are identity matrices of order $o$, $v$ and $a$ respectively. Three types of cutsets arise: $c_{o}^{\prime \prime}-$ a cutset in $g^{\prime \prime}$ having a twig from $O$

$\mathrm{c}_{\mathrm{v}}^{\prime \prime}-$ a cutset in $g^{\prime \prime}$ having a twig from $v$

$c_{a}^{\prime \prime}-$ a cutset in $g^{\prime \prime}$ having a twig from $R_{a}$

Furthermore, $D^{\prime \prime}{ }_{L}$ can also be partitioned:

$$
D^{\prime \prime}=\left[\begin{array}{lll|ll}
I_{o} & & & D^{\prime \prime}{ }_{o p} & D^{\prime \prime}{ }_{o b} \\
& I_{v} & & D^{\prime \prime}{ }_{v p} & D^{\prime \prime}{ }_{v b} \\
& & I_{a} & D^{\prime \prime}{ }_{a p} & D^{\prime \prime}{ }_{a b}
\end{array}\right]
$$

where every submatrix denoted as $D^{\prime \prime}{ }_{x y}$ is a matrix of size $x \times y$. Since $o=p, D_{o p}^{\prime \prime}$ is a square matrix. Moreover, the size of $D^{\prime \prime}{ }_{L}$ is $(o+v+a) \times(p+b) . \mathrm{KCL}$ is given as:

$$
D^{\prime \prime} i=0
$$

The partitioning above allows us to establish KCL as:

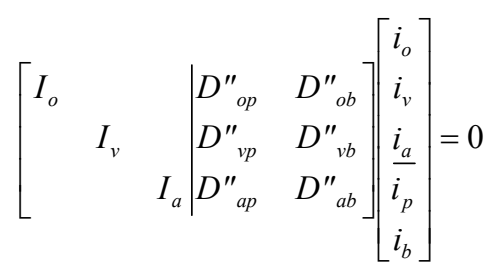

which is a system of $(o+v+b)$ cutset equations in $(o+v+a+p+b)$ branch currents. For the nullors, $i_{o}=0$, then KCL can be re-written as:

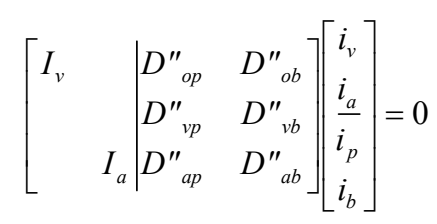

Based on the orthogonality relationship:

$$
C^{\prime \prime}{ }_{T}=D_{T}^{\prime \prime}
$$

where $D_{T}^{\prime \prime}$ stands for the transpose of $D^{\prime \prime}{ }_{T}$ (see the Figure 7), the following equalities arise:

$$
\begin{array}{lll}
C_{p o}^{\prime \prime T}=D_{o p}^{\prime \prime} & C_{v o}^{\prime \prime T}=D_{o v}^{\prime \prime} & C_{a o}^{\prime \prime T}=D_{o a}^{\prime \prime} \\
C^{\prime \prime}{ }_{p b}^{T}=D_{b p}^{\prime \prime} & C_{v b}^{\prime \prime T}=D_{b v}^{\prime \prime} & C_{a b}^{\prime \prime T}=D_{b a}^{\prime \prime}
\end{array}
$$

For the small circuit shown in Figure 6, the fundamental loop and cutset matrices are given as:

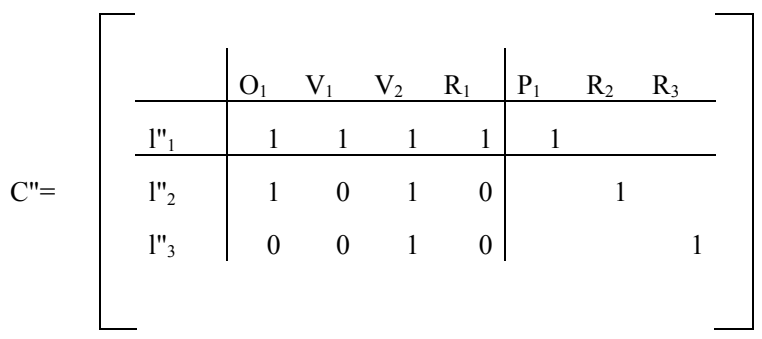




$\mathrm{D}=\left[\begin{array}{l|l|ll|l|l|ll} & \mathrm{P}_{1} & \mathrm{~V}_{1} & \mathrm{~V}_{2} & \mathrm{R}_{1} & \mathrm{O}_{1} & \mathrm{R}_{2} & \mathrm{R}_{3} \\ \hline \mathrm{c}_{1} & 1 & & & & 1 & 1 & 0 \\ \hline \mathrm{c}_{2} & & 1 & & & 1 & 0 & 0 \\ \mathrm{c}_{3} & & & 1 & & 1 & 1 & 1 \\ \hline \mathrm{c}_{4} & & & & 1 & 1 & 0 & 0\end{array}\right]$

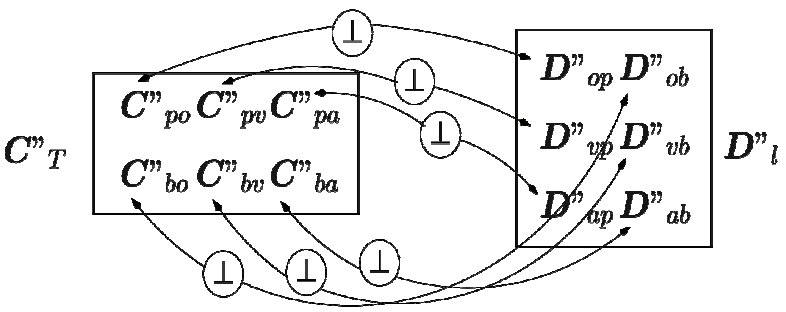

Figure 7. Orthohonality in $g^{\prime \prime}$

where the columns have been labelled with the element names and the rows with the loop and cutsets respectively. The labels however, do not belong to the matrix.

\subsection{Loop Equations of $g^{\prime}$ and $g^{\prime \prime}$}

The KVL Equations (8) and (13) are repeated here for easy reading:

$$
\begin{aligned}
& {\left[\begin{array}{lll}
C_{o p} & C_{o v} & C_{o a} \\
C_{b p} & C_{b v} & C_{b a}
\end{array} \mid I_{o} \quad I_{b}\left[\begin{array}{l}
u_{p} \\
u_{v} \\
\frac{u_{a}}{u_{o}} \\
u_{b}
\end{array}\right]=0\right.}
\end{aligned}
$$

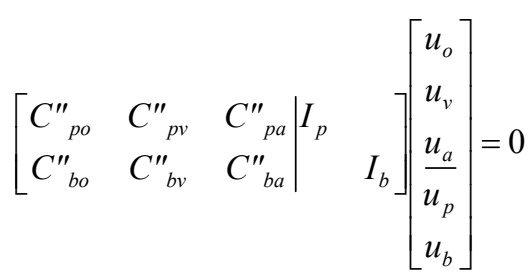

Although these equations correspond to the fundamental loops of the graphs $g^{\prime}$ and $g^{\prime \prime}$, under the selection of $t$ ' and $t$ " respectively, these equations refer in fact to the same graph and handle the same set of variables.

This means that both KVL equations contain redundant information. As can be observed schematically in the Figure 8, where the fundamental loop $l^{\prime \prime}{ }_{1}$ in $g^{\prime \prime}$ results from the combination of loops $l_{1}^{\prime \prime}$ and $l_{2}^{\prime \prime}$ in $g^{\prime}$. Therefore, it is possible to establish the next:

\section{Statement 1}

A fundamental loop in $g^{\prime}$ (resp. $g^{\prime \prime}$ ) may result from a combination of one or more fundamental loops in $g^{\prime \prime} \quad$ (resp. $g^{\prime}$ ).

\section{E. 0 A digression on the dimensions}

The considerations above lead us to define some special loops that may exist, namely:

$L^{\prime}-$ the longest loop(s) in $g^{\prime}$

$\lambda^{\prime}-$ the shortest loop(s) in $g^{\prime}$

$L^{\prime \prime}-$ the longest loop(s) in $g^{\prime \prime}$

$\lambda^{\prime \prime}-$ the shortest loop(s) in $g^{\prime \prime}$

The maximum lengths of longest loops are given as:

$$
\begin{aligned}
& \max D(L)<(p+v+a)+1 \\
& \max D\left(L^{\prime \prime}\right)<(o+v+a)+1
\end{aligned}
$$

where $\max D$ stands for "the minimum length of". Because, $o=p$ then both bounds are the same, i.e.:

$$
\max D(L)=\max D(L)=\max D\left(L^{\prime \prime}\right)<(o+v+a)+1
$$

In the case that the longest loops have the maximum length, it occurs that:

$$
L=L^{\prime \prime}
$$

i.e., a fundamental loop that appears in both graphs. The minimum lengths of the shortest loops are given by:

$$
\begin{aligned}
& \min D(\lambda)>\min (p, v, a)+1 \\
& \min D\left(\lambda^{\prime \prime}\right)>\min (o, v, a)+1
\end{aligned}
$$

where minD stands for "the minimum length of".

Because $o=p$, then both bounds are the same, i.e.:

$$
\min D(\lambda)=\min D(\lambda)=\min D\left(\lambda^{\prime \prime}\right)>\min (o, v, a)+1
$$

The minimum value for $\min (o, v, a)+1$ may be 2 , i.e., a loop formed by a parallel combination of two elements, then chances are that more than one shortest loop exist and also that a longer loop can be the result of a linear combination of several short loops. For example, these bounds are:
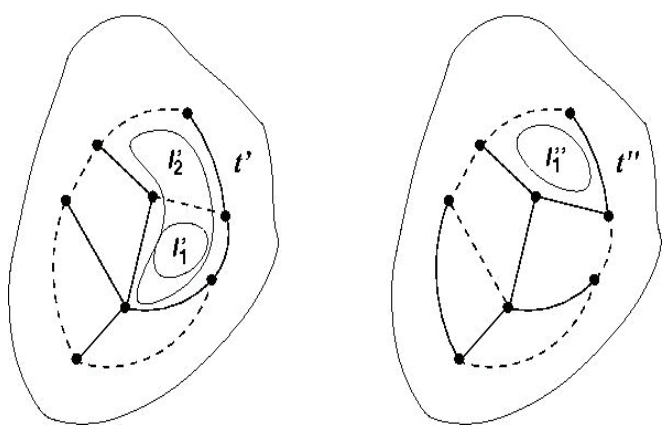

Figure 8. Redundant fundamental loops 


$$
\begin{aligned}
& \max D(L)<5 \\
& \min D(\lambda)>2
\end{aligned}
$$

and the longest loops are given as:

$$
\begin{aligned}
& L=l_{1}={ }^{\prime}\left\{P_{1}, V_{1}, V_{2}, R_{1}, O_{1}\right\} \\
& L^{\prime \prime}=l^{\prime \prime}{ }_{1}=\left\{O_{1}, V_{1}, V_{2}, R_{1}, P_{1}\right\}
\end{aligned}
$$

In addition, the shortest loops are given as:

$$
\begin{aligned}
& \lambda=I_{3}=\left\{V_{2}, R_{3}\right\} \\
& \lambda^{\prime \prime}=I^{\prime \prime}{ }_{3}=\left\{V_{2}, R_{3}\right\}
\end{aligned}
$$

i.e., in fact, the same shortest and longest loops arise in both graphs.

Because the Equations (8) and (13) refer in fact to the same graph, they can be combined in a single KVL. By re-ordering this equation according to the tree branches in $t^{\prime}$, it yields:

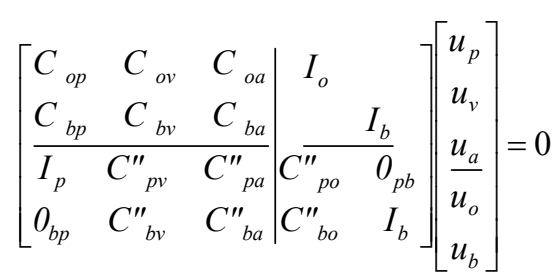

which is a system of $2 p+2 b$ loop equations, some of them being linearly dependent. Roughly speaking, some row-vectors in the equation above are wasted.

The number of linearly independent equations is given by the following:

\section{Statement 2}

The number of linearly independent loop equations is determined by:

$$
=\operatorname{Rank}\left(\left[\begin{array}{ccc|cc}
C_{o p} & C_{o v} & C_{o a} & I_{o} & \\
C_{b p} & C_{b v} & C_{b a} & & I_{b} \\
\hline I_{p} & C^{\prime \prime}{ }_{p v} & C^{\prime \prime} & C_{p a}^{\prime \prime} & O_{p b} \\
O_{b p} & C^{\prime \prime}{ }_{b v} & C^{\prime \prime} & C_{b a}^{\prime \prime} & I_{b}
\end{array}\right]\right)
$$

Besides, the linearly independent loop equations are given by the following:

\section{Statement 3}

The linearly independent loop equations are determined by:

$$
=\text { Basis }\left(\left[\begin{array}{ccc|cc}
C_{o p} & C_{o v} & C_{o a} & I_{o} & \\
C_{b p} & C_{b v} & C_{b a} & & I_{b} \\
\hline I_{p} & C^{\prime \prime}{ }_{p v} & C^{\prime \prime} & C_{p o}^{\prime \prime} & 0_{p b} \\
0_{b p} & C^{\prime \prime}{ }_{b v} & C^{\prime \prime} & C_{b a}^{\prime \prime} & C_{b o}
\end{array}\right]\right)
$$

which constitutes in fact the row space of the matrix.

For the example of the previous section, the composed matrix of the Equation (21) is given as:

$C_{\text {both }}=\left[\begin{array}{c|rrrr|rrr} & \mathrm{P}_{1} & \mathrm{~V}_{1} & \mathrm{~V}_{2} & \mathrm{R}_{1} & \mathrm{O}_{1} & \mathrm{R}_{2} & \mathrm{R}_{3} \\ \hline \mathrm{l}_{1}^{\prime} & 1 & 1 & 1 & 1 & 1 & 0 & 0 \\ \mathrm{l}_{2} & 1 & 1 & 0 & 1 & 0 & 1 & 0 \\ \mathrm{l}_{3}^{\prime} & 0 & 0 & 1 & 0 & 0 & 0 & 1 \\ \hline \mathrm{l}_{1}^{\prime \prime} & 1 & 1 & 1 & 1 & 1 & 0 & 0 \\ \mathrm{l}_{2} & 0 & 0 & 1 & 0 & 1 & 1 & 0 \\ \mathrm{l}_{3} & 0 & 0 & 1 & 0 & 0 & 0 & 1\end{array}\right]$

where the labels do not belong to the matrix. The Figure 9 shows the graph with both trees and the set of linearly independent loops. It clearly results that:

$$
l_{1}^{\prime \prime}=l_{1} \text { and } l_{3}^{\prime \prime}=l_{3}
$$

i.e., the shortest and longest loops. Therefore, the row basis of the matrix above is given as:

$\mathrm{C}_{\text {base }}=\left[\begin{array}{c|ccccccc} & \mathrm{P}_{1} & \mathrm{~V}_{1} & \mathrm{~V}_{2} & \mathrm{R}_{1} & \mathrm{O}_{1} & \mathrm{R}_{2} & \mathrm{R}_{3} \\ \hline \mathrm{l}_{1}^{\prime} & 1 & 1 & 1 & 1 & 1 & 0 & 0 \\ \mathrm{l}_{2}^{\prime} & 1 & 1 & 0 & 1 & 0 & 1 & 0 \\ \mathrm{l}_{3}^{\prime} & 0 & 0 & 1 & 0 & 0 & 0 & 1 \\ \hline 1_{2}^{\prime \prime} & 0 & 0 & 1 & 0 & 1 & 1 & 0\end{array}\right]$

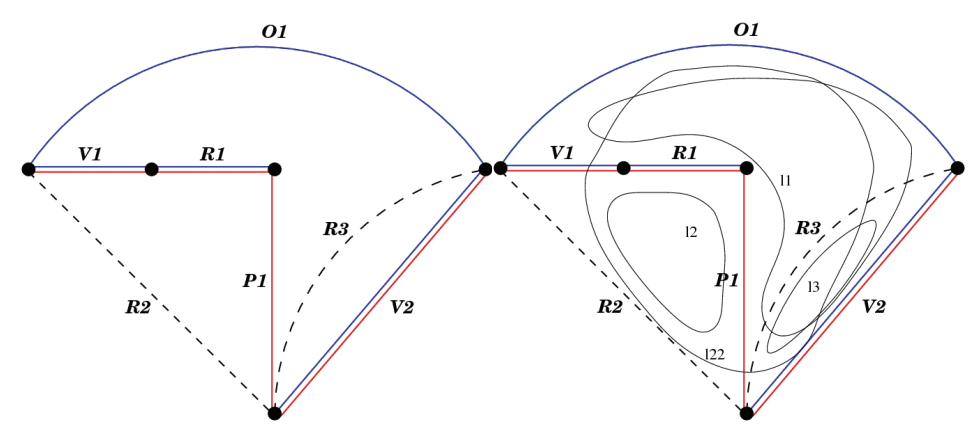

Figure 9. Combined graph \& independent loops 


\section{Conclusions}

A set of properties of the pair of conjugate trees of within the graph emanating from nonlinear resistive circuits has been presented. This approach is obtained by lookinkg at the resulting loop and cutset matrices.

\section{REFERENCES}

[1] A. F. Schwarz, "Computer-Aided Design of Microelectronic Circuits and Systems," Academic Press, Cambridge, Vol. 1, 1987.

[2] J. Vlach and K. Singhal, "Computer Methods for Circuit Analysis and Design," Van Nostrand Reinhold Company, New York, 1983.

[3] M. Hasler, "Stability of Parasitic Dynamics at a dc-
Operating Point: Topological Analysis," Proceedings of the IEEE International Symposium on Circuits and Systems, Singapore, 1991, pp. 770-773.

[4] N. Tetsuo and C. Leon O, "Topological Conditions for a Resistive Circuit Containing Negative Non-Linear Resistors to Have a Unique Solution," International Journal on Circuit Theory and Applications, Vol. 15, Vol. 2, July 1987, pp. 193-210.

[5] M. Fosseprez, M. Hasler and C. Schnetzler, "On the Number of Solutions of Piecewise-Linear Resistive Circuits," IEEE Transactions on Circuits and Systems, Vol. 18, No. 3, March 1989, pp. 393-402.

[6] M. Fossèprez and M. Hasler, "Resistive Circuit Topologies that Admit Several Solutions," International Journal on Circuit Theory and Applications, Vol. 18, No. 6, December 1990, pp. 625-638. 